\title{
Optical Properties of the Phosphorescent Trinuclear Copper(I) Complexes of Pyrazolates: Insights from Theory
}

\author{
Bo $\mathrm{Hu}^{a, b}$, Godefroid Gahungu ${ }^{a}$, Jingping Zhang ${ }^{a_{*}}$ \\ ${ }^{a}$ Faculty of Chemistry, Northeast Normal University, Changchun 130024, China \\ ${ }^{b}$ Faculty of Chemistry, Jilin Normal University, Siping 136000, China
}

\section{Supporting materials}

\section{Index}

1. Tables (S1 to S5) listing the numerical results not given in the main text

pages $2-8$

2. Figures (S1 to $\mathrm{S} 4)$ not given in the main text

pages 9-

14

\footnotetext{
* Corresponding author. Fax: +86-431-85684937; E-mail: zhangjp162@nenu.edu.cn
} 
I. Tables (S1 to S5) listing the numerical results not given in the main text

TABLE S1: TD-B3PW91/6-31G Calculated Optical Properties of Vertical- and TiltingMovement Dimers ${ }^{a}$

\begin{tabular}{|c|c|c|c|c|c|c|c|}
\hline & $\Psi_{\mathrm{v}} \rightarrow \Psi_{\mathrm{o}}$ & $\mathbf{E}_{\Psi_{\mathrm{v}}}$ & $\mathbf{E}_{\Psi_{0}}$ & $\mathbf{E}_{\mathrm{g}}$ & $\lambda_{\mathrm{em}}$ & SPE & $d_{\text {cu-cu }}$ \\
\hline \multicolumn{8}{|c|}{ Vertical-movement Dimers } \\
\hline $\mathbf{D}_{\mathrm{v} 1}$ & $\mathrm{~L} \rightarrow \mathrm{H}$ & -2.493 & -6.035 & 3.542 & 765.14 & 0.178 & 3.183 \\
\hline $\mathbf{D}_{\mathrm{v} 2}$ & $\mathrm{~L} \rightarrow \mathrm{H}$ & -2.472 & -6.075 & 3.603 & 743.14 & 0.142 & 3.239 \\
\hline $\mathbf{D}_{\mathrm{v} 3}$ & $\mathrm{~L} \rightarrow \mathrm{H}$ & -2.411 & -6.185 & 3.774 & 693.15 & 0.063 & 3.430 \\
\hline$D_{\mathrm{v} 4}$ & $\mathrm{~L}+1 \rightarrow \mathrm{H}$ & -2.364 & -6.264 & 3.900 & 665.68 & 0.021 & 3.621 \\
\hline $\mathbf{D}_{1}$ & $\mathrm{~L}+1 \rightarrow \mathrm{H}$ & -2.328 & -6.318 & 3.990 & 649.35 & 0 & 3.813 \\
\hline $\mathbf{D}_{\mathrm{v} 5}$ & $\mathrm{~L}+1 \rightarrow \mathrm{H}$ & -2.301 & -6.357 & 4.056 & 639.31 & -0.010 & 4.006 \\
\hline$D_{\mathrm{v} 6}$ & $\mathrm{~L}+1 \rightarrow \mathrm{H}$ & -2.281 & -6.385 & 4.104 & 633.05 & -0.015 & 4.200 \\
\hline $\mathbf{D}_{\mathrm{v} 7}$ & $\mathrm{~L}+1 \rightarrow \mathrm{H}$ & -2.268 & -6.406 & 4.138 & 629.03 & -0.016 & 4.394 \\
\hline \multicolumn{8}{|c|}{ Tilting-movement Dimers } \\
\hline$D_{t 1}$ & $\mathrm{~L} \rightarrow \mathrm{H}$ & -2.521 & -6.274 & 3.753 & 698.26 & 0.451 & 3.427 \\
\hline$D_{\mathrm{t} 2}$ & $\mathrm{~L}+1 \rightarrow \mathrm{H}$ & -2.471 & -6.286 & 3.815 & 686.19 & 0.263 & 3.490 \\
\hline $\mathbf{D}_{\mathbf{t 3}}$ & $\mathrm{L}+1 \rightarrow \mathrm{H}$ & -2.417 & -6.298 & 3.881 & 671.03 & 0.108 & 3.590 \\
\hline$D_{t 4}$ & $\mathrm{~L}+1 \rightarrow \mathrm{H}$ & -2.370 & -6.308 & 3.938 & 659.36 & 0.034 & 3.698 \\
\hline $\mathbf{D}_{1}$ & $\mathrm{~L}+1 \rightarrow \mathrm{H}$ & -2.328 & -6.318 & 3.990 & 649.35 & 0 & 3.813 \\
\hline$D_{t 5}$ & $\mathrm{~L}+1 \rightarrow \mathrm{H}$ & -2.291 & -6.331 & 4.040 & 641.12 & -0.014 & 3.936 \\
\hline$D_{\text {t6 }}$ & $\mathrm{L}+1 \rightarrow \mathrm{H}$ & -2.263 & -6.346 & 4.083 & 634.96 & -0.019 & 4.064 \\
\hline$D_{\mathbf{t 7}}$ & $\mathrm{L}+1 \rightarrow \mathrm{H}$ & -2.241 & -6.360 & 4.119 & 630.02 & -0.020 & 4.198 \\
\hline
\end{tabular}


TABLE S2: Natural Atomic Orbital Populations of the Lowest-lying Triplets Excited-states for the Emission of Moved Dimers, as Provided by CIS/6-31G ${ }^{a}$

\begin{tabular}{|c|c|c|c|c|c|c|c|c|}
\hline \multirow[b]{2}{*}{ Complex } & \multicolumn{3}{|c|}{$\mathbf{C u}$} & \multicolumn{3}{|c|}{$\mathrm{Cu}$} & \multirow[b]{2}{*}{$\sum \Delta \mathbf{C u}$} & \multirow[b]{2}{*}{$\sum \Delta \mathbf{L}$} \\
\hline & $4 s$ & $4 p$ & 3d & $4 s$ & $4 p$ & 3d & & \\
\hline \multicolumn{9}{|c|}{ Vertical-movement Dimers } \\
\hline $\mathbf{D}_{\mathrm{v} 1}$ & & & & 0.986 & 0.146 & 8.901 & -0.163 & 0.171 \\
\hline$D_{\mathrm{v} 2}$ & & & & 0.979 & 0.143 & 8.916 & -0.161 & 0.168 \\
\hline$D_{\mathrm{v} 3}$ & 0.538 & 0.063 & 9.584 & 0.943 & 0.132 & 8.985 & -0.154 & 0.160 \\
\hline$D_{\mathrm{v} 4}$ & 0.765 & 0.091 & 9.263 & 0.786 & 0.087 & 9.253 & -0.157 & 0.161 \\
\hline$D_{\mathrm{v} 5}$ & 0.783 & 0.083 & 9.258 & 0.802 & 0.082 & 9.246 & -0.143 & 0.147 \\
\hline$D_{\mathrm{v} 6}$ & 0.789 & 0.080 & 9.258 & 0.807 & 0.080 & 9.242 & -0.138 & 0.142 \\
\hline$D_{\mathrm{v} 7}$ & 0.793 & 0.078 & 9.257 & 0.811 & 0.079 & 9.241 & -0.134 & 0.138 \\
\hline \multicolumn{9}{|c|}{ Parallel-movement Dimers } \\
\hline $\mathbf{D}_{\mathrm{p} 1}$ & 0.771 & 0.082 & 9.285 & 0.821 & 0.091 & 9.211 & -0.129 & 0.133 \\
\hline$D_{p 2}$ & 0.766 & 0.080 & 9.291 & 0.829 & 0.092 & 9.201 & -0.124 & 0.128 \\
\hline$D_{p 3}$ & 0.788 & 0.083 & 9.257 & 0.805 & 0.086 & 9.237 & -0.129 & 0.133 \\
\hline$D_{p 4}$ & 0.797 & 0.086 & 9.239 & 0.788 & 0.084 & 9.260 & -0.140 & 0.144 \\
\hline$D_{p 5}$ & 0.763 & 0.085 & 9.275 & 0.803 & 0.084 & 9.238 & -0.154 & 0.158 \\
\hline$D_{p 6}$ & 0.752 & 0.082 & 9.290 & 0.815 & 0.084 & 9.224 & -0.158 & 0.162 \\
\hline \multicolumn{9}{|c|}{ Rotational Dimers } \\
\hline$D_{60}$ & 0.826 & 0.084 & 9.209 & 0.772 & 0.077 & 9.290 & -0.147 & 0.151 \\
\hline $\mathbf{D}_{120}$ & 0.928 & 0.115 & 9.024 & 0.637 & 0.062 & 9.493 & -0.163 & 0.168 \\
\hline$D_{180}$ & 0.819 & 0.079 & 9.231 & 0.779 & 0.075 & 9.278 & -0.177 & 0.181 \\
\hline $\mathbf{D}_{240}$ & 0.880 & 0.097 & 9.119 & 0.662 & 0.075 & 9.413 & -0.158 & 0.163 \\
\hline $\mathbf{D}_{\mathbf{3 0 0}}$ & 0.785 & 0.078 & 9.271 & 0.819 & 0.082 & 9.226 & -0.143 & 0.147 \\
\hline \multicolumn{9}{|c|}{ Tilting-movement Dimers } \\
\hline $\mathbf{D}_{\mathrm{t} 1}$ & 0.528 & 0.064 & 9.596 & 0.957 & 0.134 & 8.965 & -0.158 & 0.165 \\
\hline$D_{t 2}$ & 0.560 & 0.065 & 9.556 & 0.938 & 0.128 & 8.998 & -0.154 & 0.159 \\
\hline $\mathbf{D}_{\mathbf{t} 3}$ & 0.771 & 0.094 & 9.253 & 0.775 & 0.089 & 9.263 & -0.161 & 0.165 \\
\hline
\end{tabular}




\begin{tabular}{lllllllll}
\hline $\mathbf{D}_{\mathbf{t} 4}$ & 0.774 & 0.090 & 9.255 & 0.785 & 0.086 & 9.257 & -0.154 & 0.158 \\
$\mathbf{D}_{\mathbf{t} 5}$ & 0.778 & 0.083 & 9.262 & 0.802 & 0.083 & 9.244 & -0.146 & 0.149 \\
$\mathbf{D}_{\mathbf{t} 6}$ & 0.781 & 0.080 & 9.264 & 0.808 & 0.081 & 9.239 & -0.143 & 0.147 \\
$\mathbf{D}_{\mathbf{t} 7}$ & 0.784 & 0.078 & 9.265 & 0.812 & 0.080 & 9.237 & -0.141 & 0.144
\end{tabular}

${ }^{a} \sum \Delta \mathrm{Cu}$ and $\sum \Delta \mathrm{L}=$ the differences of $\mathrm{Cu}(\mathrm{I})$ centers, organic ligands between the lowest-lying triplet excited-state and ground state in the natural atomic orbital populations, respectively. The negative value denotes the increases of orbital population in the electronic transition from the lowest-lying triplet excited-state to the ground state. Natural atomic populations of the ground states for the moved dimers listed in Table 3 in the main text. 
TABLE S3: The Maximum Emission Wavelength $\left(\lambda_{\mathrm{em}}, \mathbf{n m}\right)$ for the Various Triplet ExcitedState Conformations as Function of Calculation Method and Basis Set

\begin{tabular}{|c|c|c|}
\hline Complex & TD-B3P86/6-31G & TD-B3LYP/6-31G \\
\hline \multicolumn{3}{|c|}{ Monomer } \\
\hline $\mathbf{M}$ & 607.57 & 597.14 \\
\hline \multicolumn{3}{|c|}{ Pristine Dimers } \\
\hline $\mathbf{D}_{1}$ & 642.30 & 629.83 \\
\hline $\mathbf{D}_{2}$ & 608.03 & 595.06 \\
\hline \multicolumn{3}{|c|}{ Vertical-movement Dimers } \\
\hline $\mathbf{D}_{\mathrm{v} 1}$ & 754.02 & 731.65 \\
\hline$D_{\mathrm{v} 2}$ & 732.75 & 712.46 \\
\hline$D_{\mathrm{v} 3}$ & 684.50 & 668.60 \\
\hline$D_{\mathrm{v} 4}$ & 658.07 & 644.36 \\
\hline$D_{\mathrm{v} 5}$ & 632.68 & 620.93 \\
\hline$D_{\mathrm{v} 6}$ & 626.84 & 615.53 \\
\hline $\mathbf{D}_{\mathrm{v} 7}$ & 623.14 & 612.00 \\
\hline \multicolumn{3}{|c|}{ Parallel-movement Dimers } \\
\hline $\mathbf{D}_{\mathrm{p} 1}$ & 634.64 & 618.06 \\
\hline $\mathbf{D}_{\mathbf{p} 2}$ & 634.06 & 617.79 \\
\hline $\mathbf{D}_{\mathrm{p} 3}$ & 630.10 & 614.63 \\
\hline $\mathbf{D}_{\mathrm{p} 4}$ & 633.15 & 618.47 \\
\hline$D_{p 5}$ & 640.92 & 629.37 \\
\hline $\mathbf{D}_{\mathrm{p} 6}$ & 631.78 & 621.32 \\
\hline \multicolumn{3}{|c|}{ Rotational Dimers } \\
\hline$D_{60}$ & 618.70 & 605.65 \\
\hline $\mathbf{D}_{120}$ & 619.70 & 606.71 \\
\hline $\mathbf{D}_{180}$ & 583.38 & 571.16 \\
\hline $\mathbf{D}_{\mathbf{2 4 0}}$ & 621.34 & 609.29 \\
\hline $\mathbf{D}_{300}$ & 615.71 & 602.58 \\
\hline \multicolumn{3}{|c|}{ Tilting-movement Dimers } \\
\hline$D_{t 1}$ & 688.41 & 670.05 \\
\hline
\end{tabular}




\begin{tabular}{lll}
\hline $\mathbf{D}_{\mathfrak{t} 2}$ & 676.91 & 660.13 \\
$\mathbf{D}_{\mathfrak{t} 3}$ & 662.59 & 647.73 \\
$\mathbf{D}_{\mathbf{t} 4}$ & 651.72 & 638.18 \\
$\mathbf{D}_{\mathbf{t} 5}$ & 634.56 & 622.97 \\
$\mathbf{D}_{\mathbf{t} 6}$ & 628.90 & 617.89 \\
$\mathbf{D}_{\mathbf{t}}$ & 624.28 & 613.60
\end{tabular}


TABLE S4: TD-B3P86/6-31G Calculated Optical Properties of Vertical- and Tilting-Movement Dimers $^{a}$

\begin{tabular}{|c|c|c|c|c|c|c|c|}
\hline & $\Psi_{v} \rightarrow \Psi_{0}$ & $\overline{\mathbf{E}_{\boldsymbol{\Psi}_{\mathrm{v}}}}$ & $\mathbf{E}_{\Psi_{0}}$ & $\mathbf{E}_{\mathrm{g}}$ & $\lambda_{\mathrm{em}}$ & $\overline{\text { SPE }}$ & $d_{\mathrm{cu}-\mathrm{cu}}$ \\
\hline \multicolumn{8}{|c|}{ Vertical-movement Dimers } \\
\hline$D_{\mathrm{v} 1}$ & $\mathrm{~L} \rightarrow \mathrm{H}$ & -3.091 & -6.649 & 3.558 & 754.02 & 0.163 & 3.183 \\
\hline$D_{\mathrm{v} 2}$ & $\mathrm{~L} \rightarrow \mathrm{H}$ & -3.071 & -6.689 & 3.618 & 732.75 & 0.129 & 3.239 \\
\hline $\mathbf{D}_{\mathrm{v} 3}$ & $\mathrm{~L} \rightarrow \mathrm{H}$ & -3.015 & -6.801 & 3.786 & 684.50 & 0.055 & 3.430 \\
\hline $\mathbf{D}_{\mathrm{v} 4}$ & $\mathrm{~L}+1 \rightarrow \mathrm{H}$ & -2.966 & -6.882 & 3.916 & 658.07 & 0.017 & 3.621 \\
\hline $\mathbf{D}_{1}$ & $\mathrm{~L}+1 \rightarrow \mathrm{H}$ & -2.934 & -6.938 & 4.004 & 642.30 & 0 & 3.813 \\
\hline$D_{\mathrm{v} 5}$ & $\mathrm{~L}+1 \rightarrow \mathrm{H}$ & -2.910 & -6.978 & 4.068 & 632.68 & -0.007 & 4.006 \\
\hline$D_{\mathrm{v} 6}$ & $\mathrm{~L}+1 \rightarrow \mathrm{H}$ & -2.893 & -7.008 & 4.115 & 626.84 & -0.009 & 4.200 \\
\hline$D_{\mathrm{v} 7}$ & $\mathrm{~L}+1 \rightarrow \mathrm{H}$ & -2.883 & -7.029 & 4.146 & 623.14 & -0.009 & 4.394 \\
\hline \multicolumn{8}{|c|}{ Tilting-movement Dimers } \\
\hline$D_{t 1}$ & $\mathrm{~L} \rightarrow \mathrm{H}$ & -3.118 & -6.889 & 3.771 & 688.41 & 0.435 & 3.427 \\
\hline$D_{12}$ & $\mathrm{~L}+1 \rightarrow \mathrm{H}$ & -3.075 & -6.902 & 3.827 & 676.91 & 0.251 & 3.490 \\
\hline $\mathbf{D}_{\mathfrak{t} 3}$ & $\mathrm{~L}+1 \rightarrow \mathrm{H}$ & -3.021 & -6.915 & 3.894 & 662.59 & 0.101 & 3.590 \\
\hline$D_{t 4}$ & $\mathrm{~L}+1 \rightarrow \mathrm{H}$ & -2.975 & -6.926 & 3.951 & 651.72 & 0.030 & 3.698 \\
\hline $\mathbf{D}_{1}$ & $\mathrm{~L}+1 \rightarrow \mathrm{H}$ & -2.934 & -6.938 & 4.004 & 642.30 & 0 & 3.813 \\
\hline$D_{t 5}$ & $\mathrm{~L}+1 \rightarrow \mathrm{H}$ & -2.899 & -6.952 & 4.053 & 634.56 & -0.011 & 3.936 \\
\hline$D_{t 6}$ & $\mathrm{~L}+1 \rightarrow \mathrm{H}$ & -2.873 & -6.968 & 4.095 & 628.90 & -0.014 & 4.064 \\
\hline$D_{\mathbf{t} 7}$ & $\mathrm{~L}+1 \rightarrow \mathrm{H}$ & -2.854 & -6.983 & 4.129 & 624.28 & -0.014 & 4.198 \\
\hline $\begin{array}{l}{ }^{a} \Psi \mathrm{o}= \\
\text { involvec } \\
\text { emitting } \\
\text { lowest-ly }\end{array}$ & $\begin{array}{l}\text { and } \Psi_{\mathrm{v}}= \\
\text { main em } \\
\text { ion. } \lambda_{\mathrm{em}} \\
\text { t excited- }\end{array}$ & $\begin{array}{l}\text { lorbita } \\
\text { trans } \\
\text { maximı } \\
\text { cu-cu }=\end{array}$ & $\begin{array}{l}\text { define t } \\
\text { espectiv } \\
\text { sion w } \\
\text { rtest int }\end{array}$ & $\begin{array}{l}\text { transi } \\
\text { the }\end{array}$ & $\begin{array}{l}\mathrm{v} \text { and } \mathrm{E} \\
\text { gap for }\end{array}$ & $\begin{array}{l}\text { energie } \\
\text { s invol } \\
\text { nt eners } \\
\text { HOMO }\end{array}$ & $\begin{array}{l}\text { or FM } \\
\text { the m } \\
\text { ee) of } \\
\text { MO. }\end{array}$ \\
\hline
\end{tabular}


TABLE S5: TD-B3LYP/6-31G Calculated Optical Properties of Vertical- and Tilting-Movement Dimers $^{a}$

\begin{tabular}{|c|c|c|c|c|c|c|c|}
\hline & $\Psi_{\mathrm{v}} \rightarrow \Psi_{0}$ & $\mathbf{E}_{\boldsymbol{\Psi v}_{\mathrm{v}}}$ & $\mathbf{E}_{\Psi_{0}}$ & $\mathbf{E}_{\mathrm{g}}$ & $\lambda_{\mathrm{em}}$ & SPE & $d_{\text {cu-cu }}$ \\
\hline \multicolumn{8}{|c|}{ Vertical-movement Dimers } \\
\hline $\mathbf{D}_{\mathrm{v} 1}$ & $\mathrm{~L} \rightarrow \mathrm{H}$ & -2.433 & -6.010 & 3.577 & 731.65 & 0.184 & 3.183 \\
\hline $\mathbf{D}_{\mathrm{v} 2}$ & $\mathrm{~L} \rightarrow \mathrm{H}$ & -2.414 & -6.052 & 3.638 & 712.46 & 0.147 & 3.239 \\
\hline $\mathbf{D}_{\mathrm{v} 3}$ & $\mathrm{~L} \rightarrow \mathrm{H}$ & -2.365 & -6.168 & 3.803 & 668.60 & 0.064 & 3.430 \\
\hline $\mathbf{D}_{\mathrm{v} 4}$ & $\mathrm{~L}+1 \rightarrow \mathrm{H}$ & -2.314 & -6.252 & 3.938 & 644.36 & 0.021 & 3.621 \\
\hline $\mathbf{D}_{1}$ & $\mathrm{~L}+1 \rightarrow \mathrm{H}$ & -2.287 & -6.310 & 4.023 & 629.83 & 0 & 3.813 \\
\hline $\mathbf{D}_{\mathrm{v} 5}$ & $\mathrm{~L}+1 \rightarrow \mathrm{H}$ & -2.267 & -6.354 & 4.087 & 620.93 & -0.009 & 4.006 \\
\hline $\mathbf{D}_{\mathrm{v} 6}$ & $\mathrm{~L}+1 \rightarrow \mathrm{H}$ & -2.254 & -6.387 & 4.133 & 615.53 & -0.011 & 4.200 \\
\hline $\mathbf{D}_{\mathrm{v} 7}$ & $\mathrm{~L}+1 \rightarrow \mathrm{H}$ & -2.247 & -6.411 & 4.164 & 612.00 & -0.011 & 4.394 \\
\hline \multicolumn{8}{|c|}{ Tilting-movement Dimers } \\
\hline$D_{t 1}$ & $\mathrm{~L} \rightarrow \mathrm{H}$ & -2.457 & -6.255 & 3.798 & 670.05 & 0.460 & 3.427 \\
\hline$D_{t 2}$ & $\mathrm{~L}+1 \rightarrow \mathrm{H}$ & -2.420 & -6.270 & 3.850 & 660.13 & 0.269 & 3.490 \\
\hline $\mathbf{D}_{\mathbf{t} 3}$ & $\mathrm{~L}+1 \rightarrow \mathrm{H}$ & -2.369 & -6.284 & 3.915 & 647.73 & 0.110 & 3.590 \\
\hline$D_{t 4}$ & $\mathrm{~L}+1 \rightarrow \mathrm{H}$ & -2.325 & -6.297 & 3.972 & 638.18 & 0.034 & 3.698 \\
\hline $\mathbf{D}_{1}$ & $\mathrm{~L}+1 \rightarrow \mathrm{H}$ & -2.287 & -6.310 & 4.023 & 629.83 & 0 & 3.813 \\
\hline$D_{t 5}$ & $\mathrm{~L}+1 \rightarrow \mathrm{H}$ & -2.254 & -6.326 & 4.072 & 622.97 & -0.013 & 3.936 \\
\hline$D_{\text {t6 }}$ & $\mathrm{L}+1 \rightarrow \mathrm{H}$ & -2.230 & -6.345 & 4.115 & 617.89 & -0.016 & 4.064 \\
\hline$D_{\mathbf{t 7}}$ & $\mathrm{L}+1 \rightarrow \mathrm{H}$ & -2.214 & -6.363 & 4.149 & 613.60 & -0.016 & 4.198 \\
\hline $\begin{array}{l}{ }^{a} \Psi \mathrm{Yo}= \\
\text { involvec } \\
\text { emitting }\end{array}$ & $\begin{array}{l}\text { and } \Psi_{\mathrm{v}} \\
\text { main er } \\
\text { tion. } \lambda_{\text {em }} \\
\text { t excited }\end{array}$ & $\begin{array}{l}\text { lorbita } \\
\text { trans }\end{array}$ & $\begin{array}{l}\text { define th } \\
\text { espective } \\
\text { ssion wa }\end{array}$ & $\begin{array}{l}\text { transiti } \\
=\text { the el } \\
\text { h }(\mathrm{nm}) .\end{array}$ & $\begin{array}{l}\text { gap for } \mathrm{E}_{\Psi} \\
\text { gap for }\end{array}$ & $\begin{array}{l}\text { energie } \\
\text { s invol } \\
\text { int ener }\end{array}$ & $\begin{array}{l}\text { or FM } \\
\text { the mi } \\
\text { ee) of }\end{array}$ \\
\hline
\end{tabular}


II. Figures (S1 to S4) not given in the main text
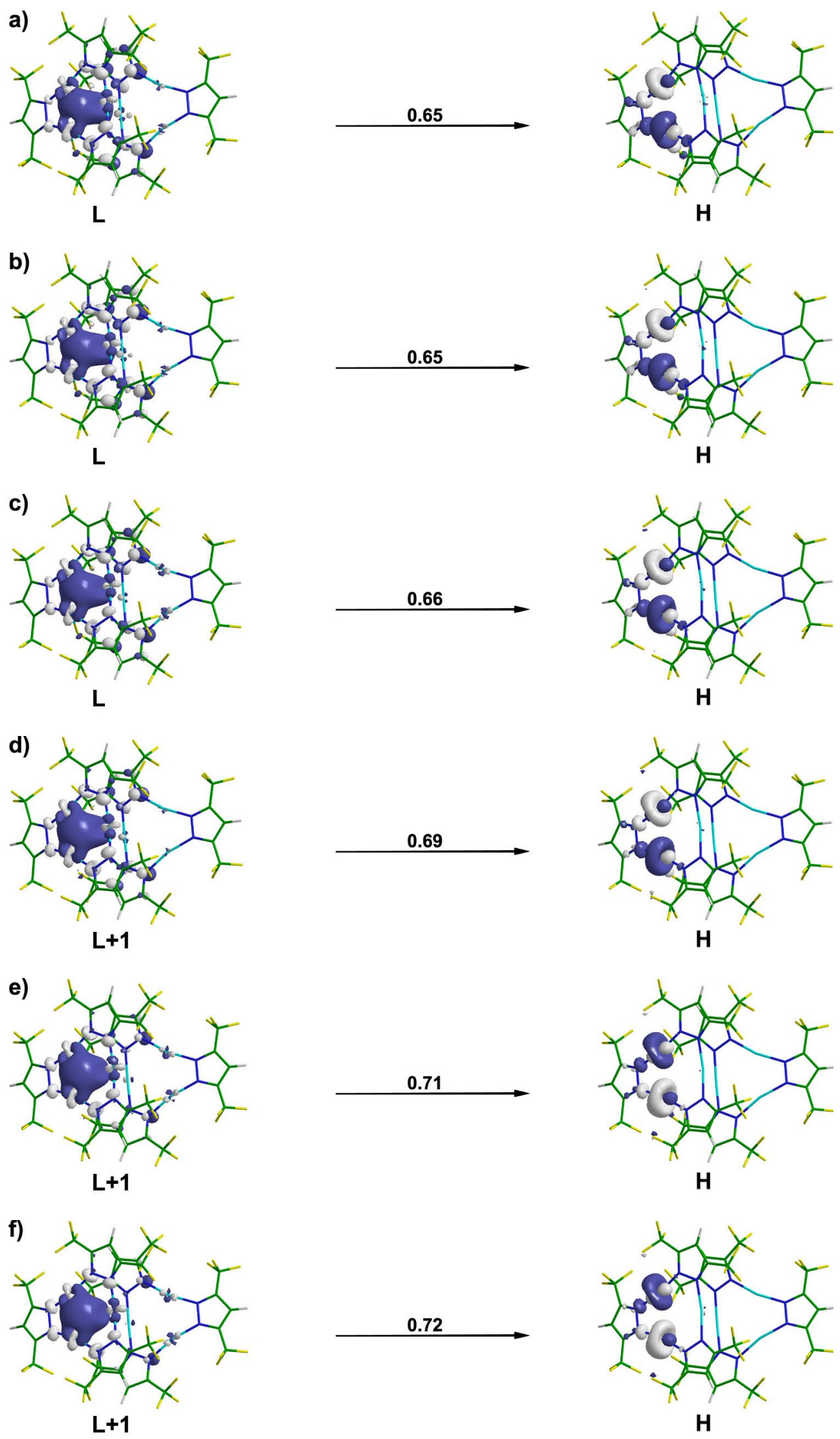

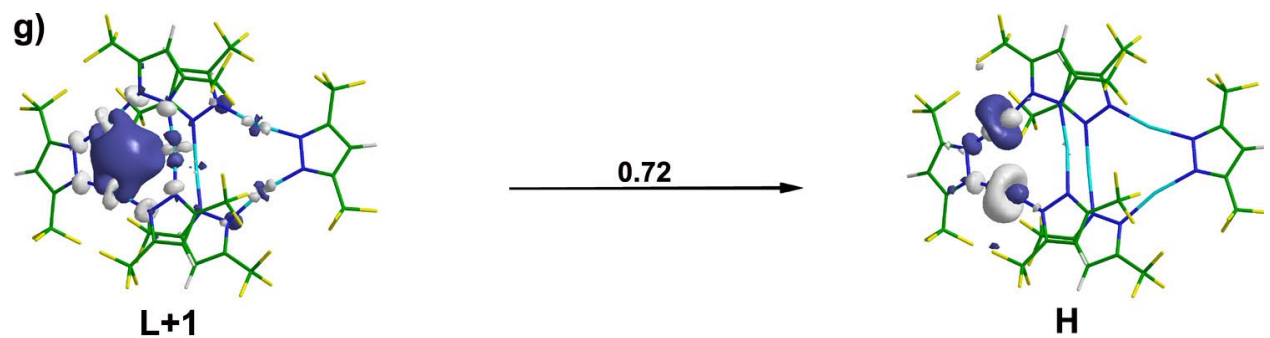

Figure S1. The TD-B3PW91/6-31G calculated triplet electron transition (illustrations) for a) $D_{v 1}, b$ ) $D_{v 2}$, c) $D_{v 3}$, d) $D_{v 4}$, e) $D_{v 5}$, f) $D_{v 6}$, g) $D_{v 7}$.

a)
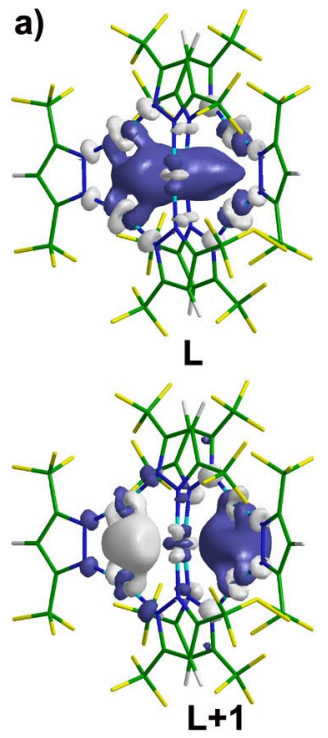

b)

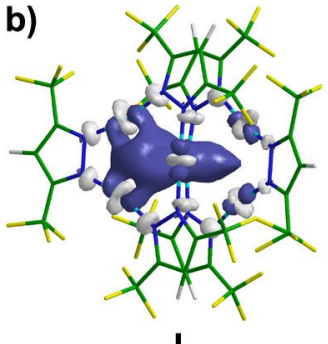

$\mathbf{L}$

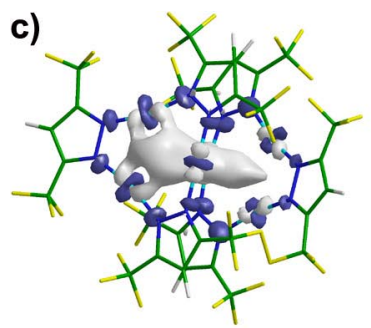

$\mathbf{L}$

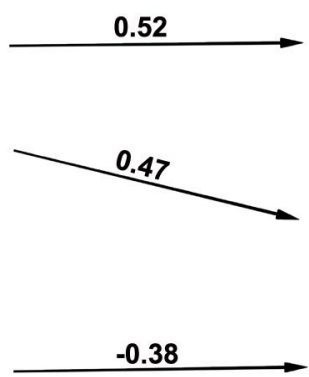

0.69

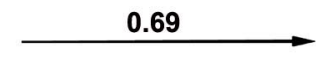

0.64

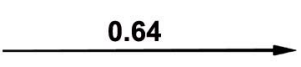

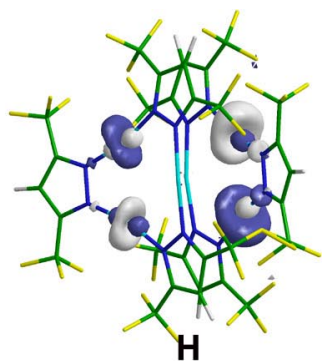
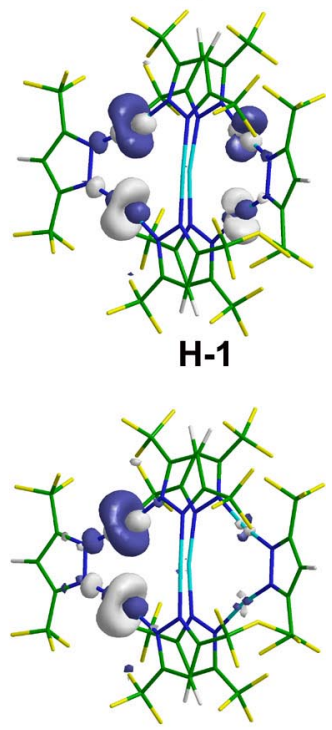

$\mathrm{H}-1$

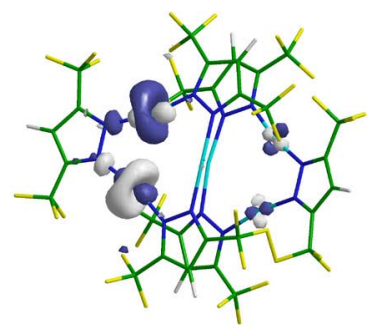

$\mathrm{H}-2$ 
d)

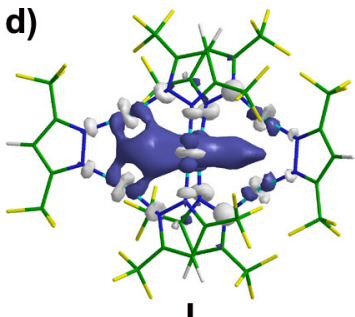

$\mathbf{L}$
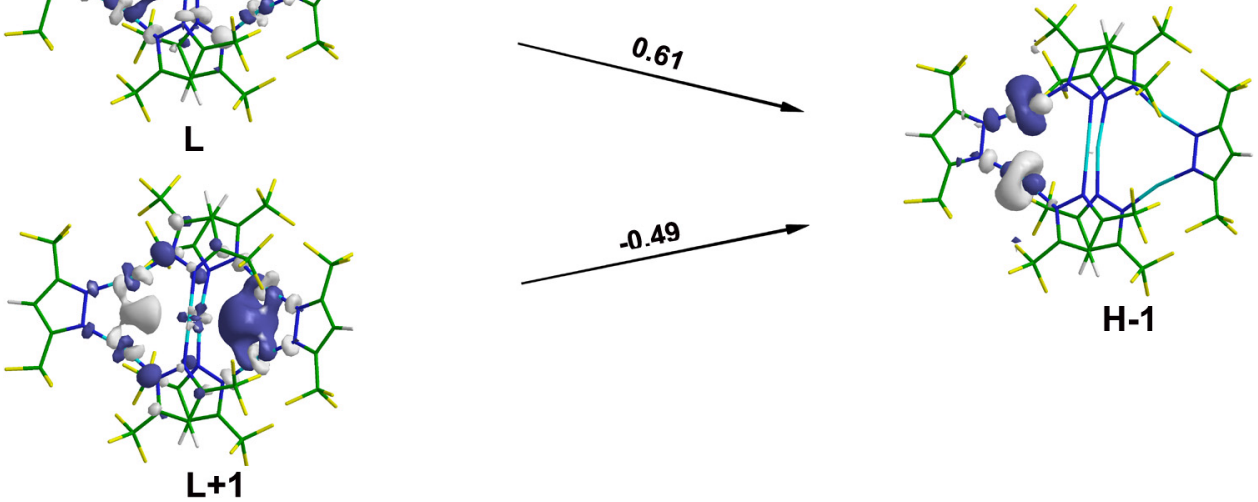

H-1

e)
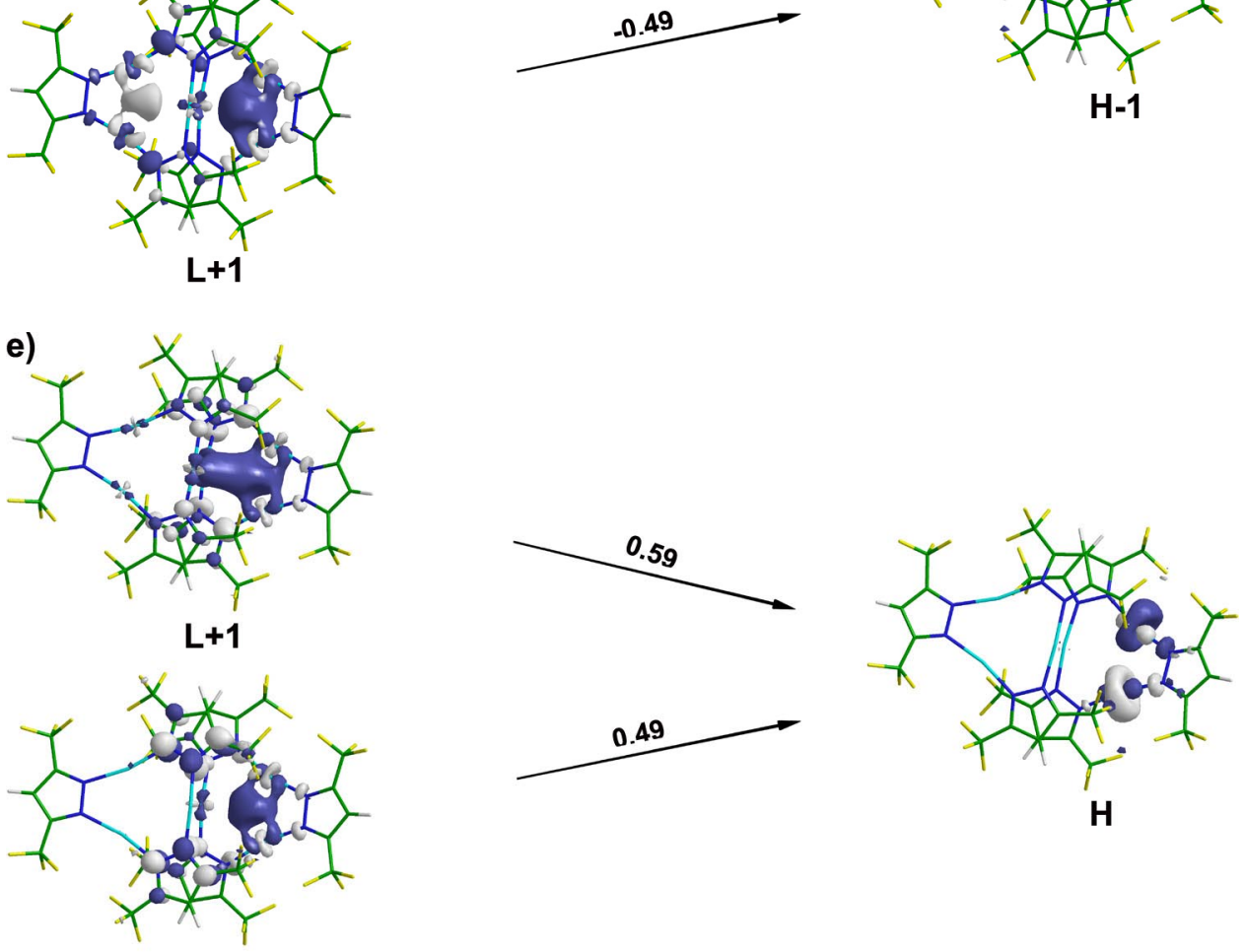

L+2

f)

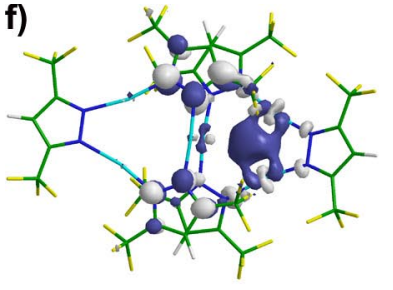

L+2
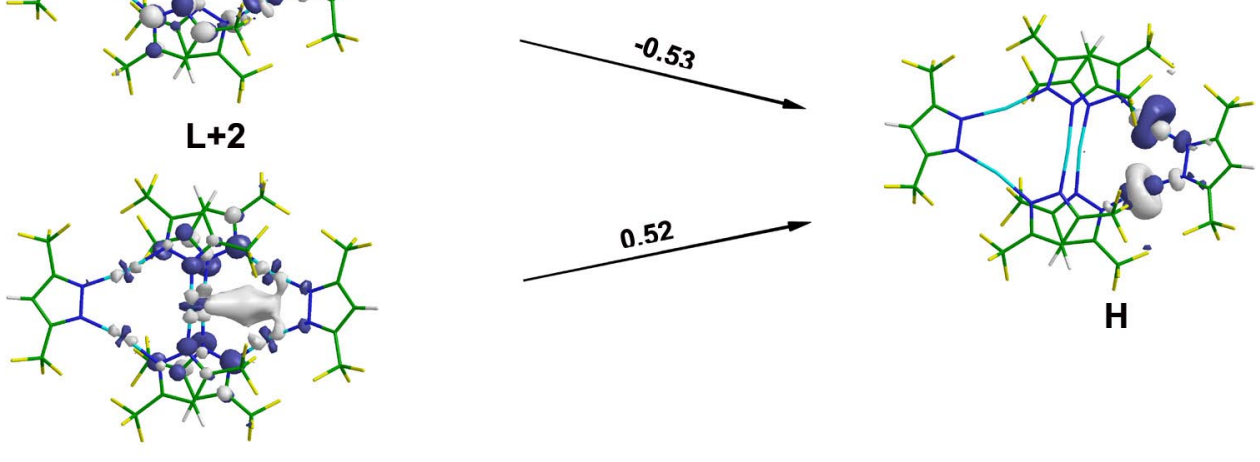

L+1

Figure S2. The TD-B3PW91/6-31G calculated triplet electron transition (illustrations) for a) $\mathrm{D}_{\mathrm{p} 1}$, b) $D_{\mathrm{p} 2}$, c) $D_{\mathrm{p} 3}$, d) $\left.\mathrm{D}_{\mathrm{p} 4}, \mathrm{e}\right) \mathrm{D}_{\mathrm{p} 5}$, f) $\mathrm{D}_{\mathrm{p} 6}$. 
a)
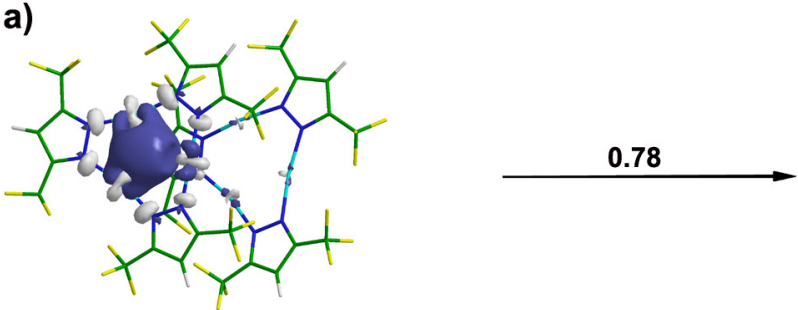

L+1

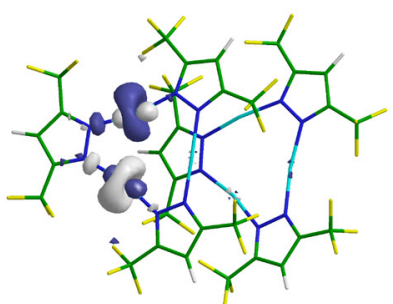

H

b)
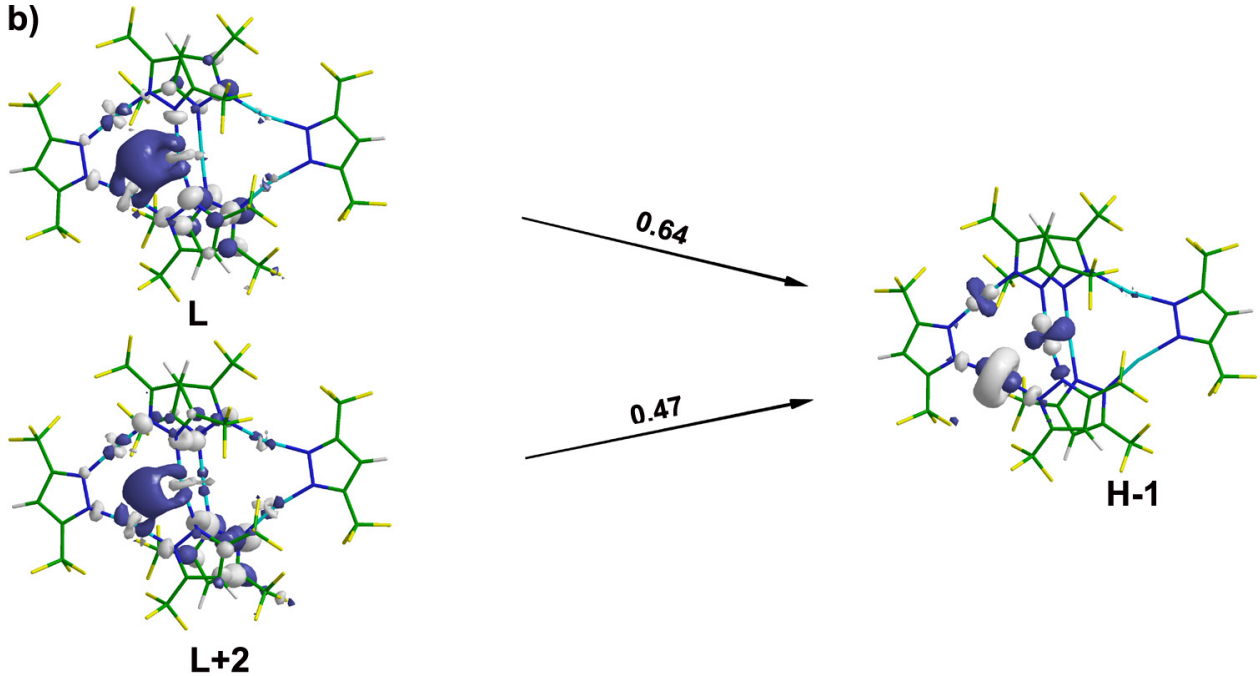

H-1

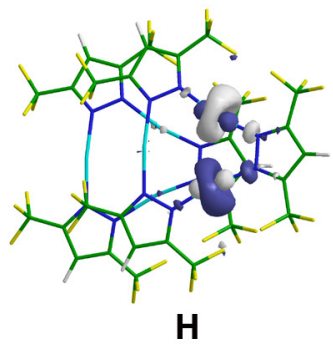

d)
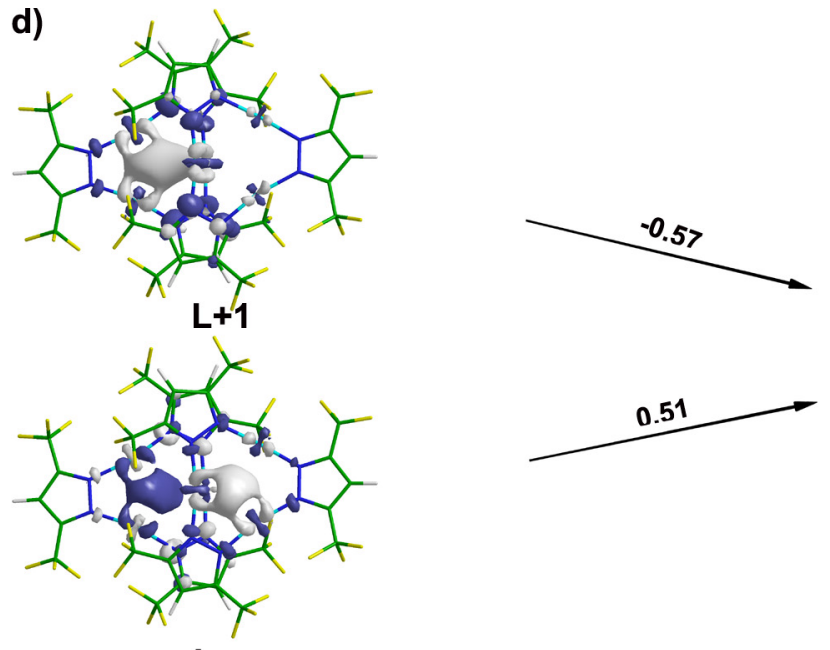
e)
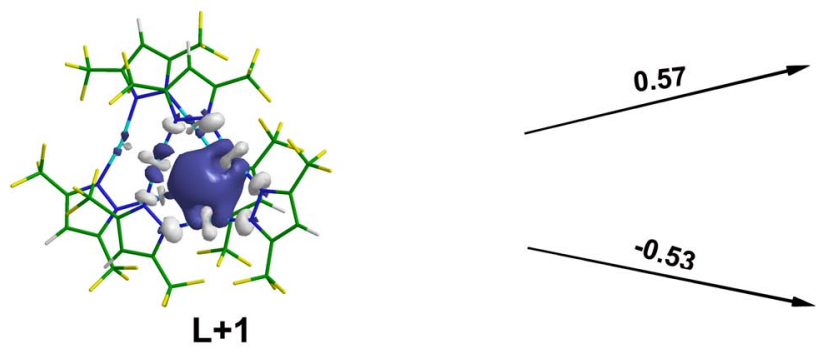

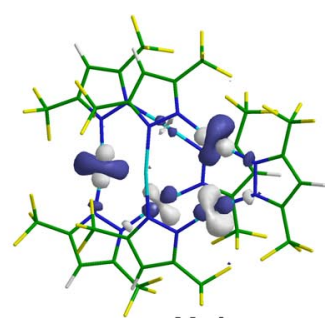

$\mathrm{H}-1$

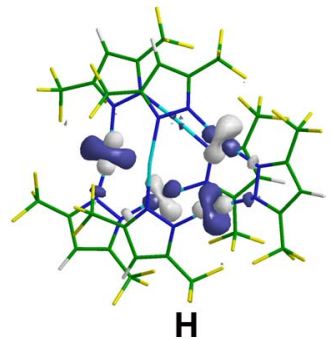

Figure S3. The TD-B3PW91/6-31G calculated triplet electron transition (illustrations) for a) $\mathrm{D}_{60}$, b) $\mathrm{D}_{120}$, c) $\mathrm{D}_{180}$, d) $\mathrm{D}_{240}$, e) $\mathrm{D}_{300}$.
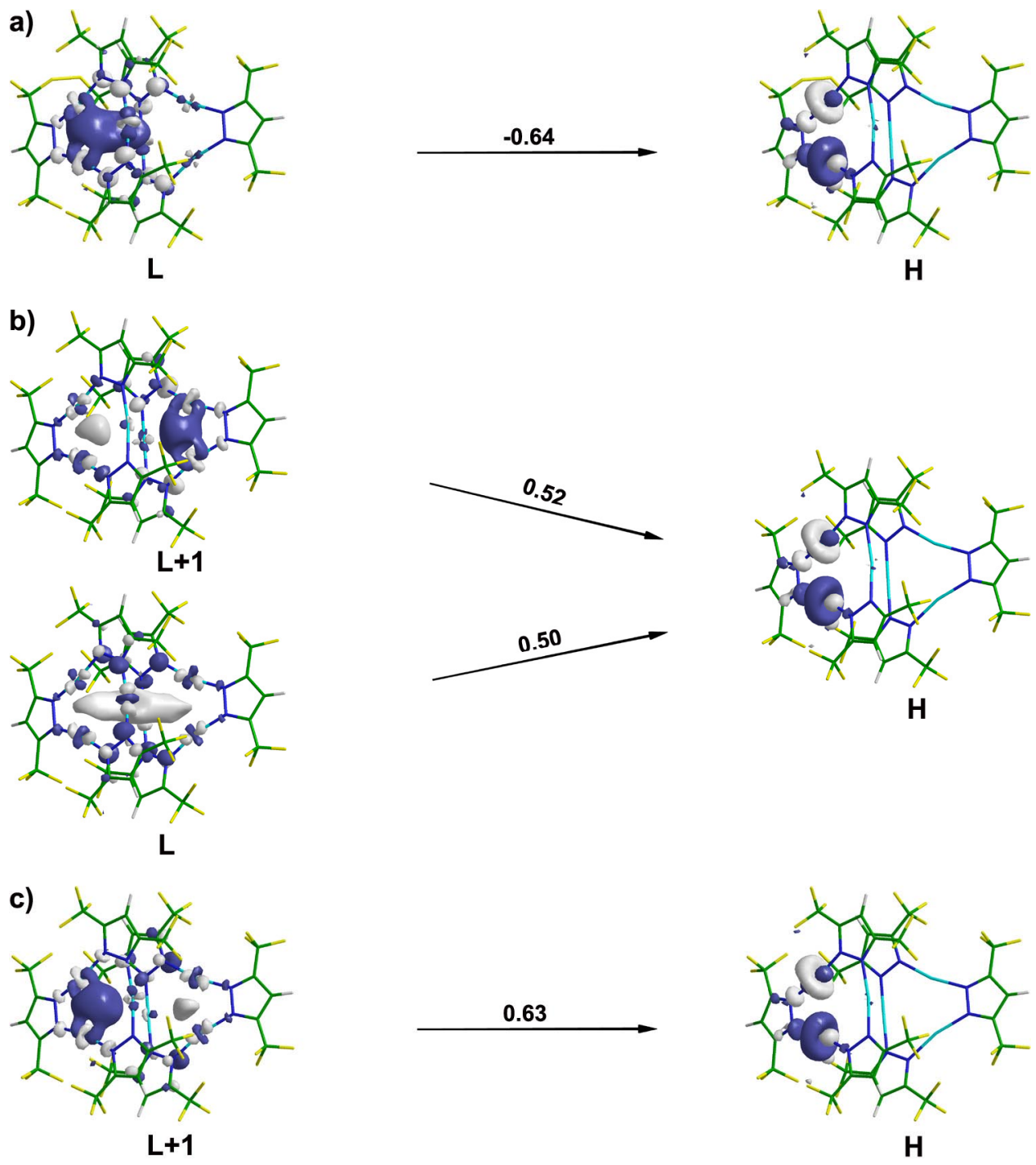

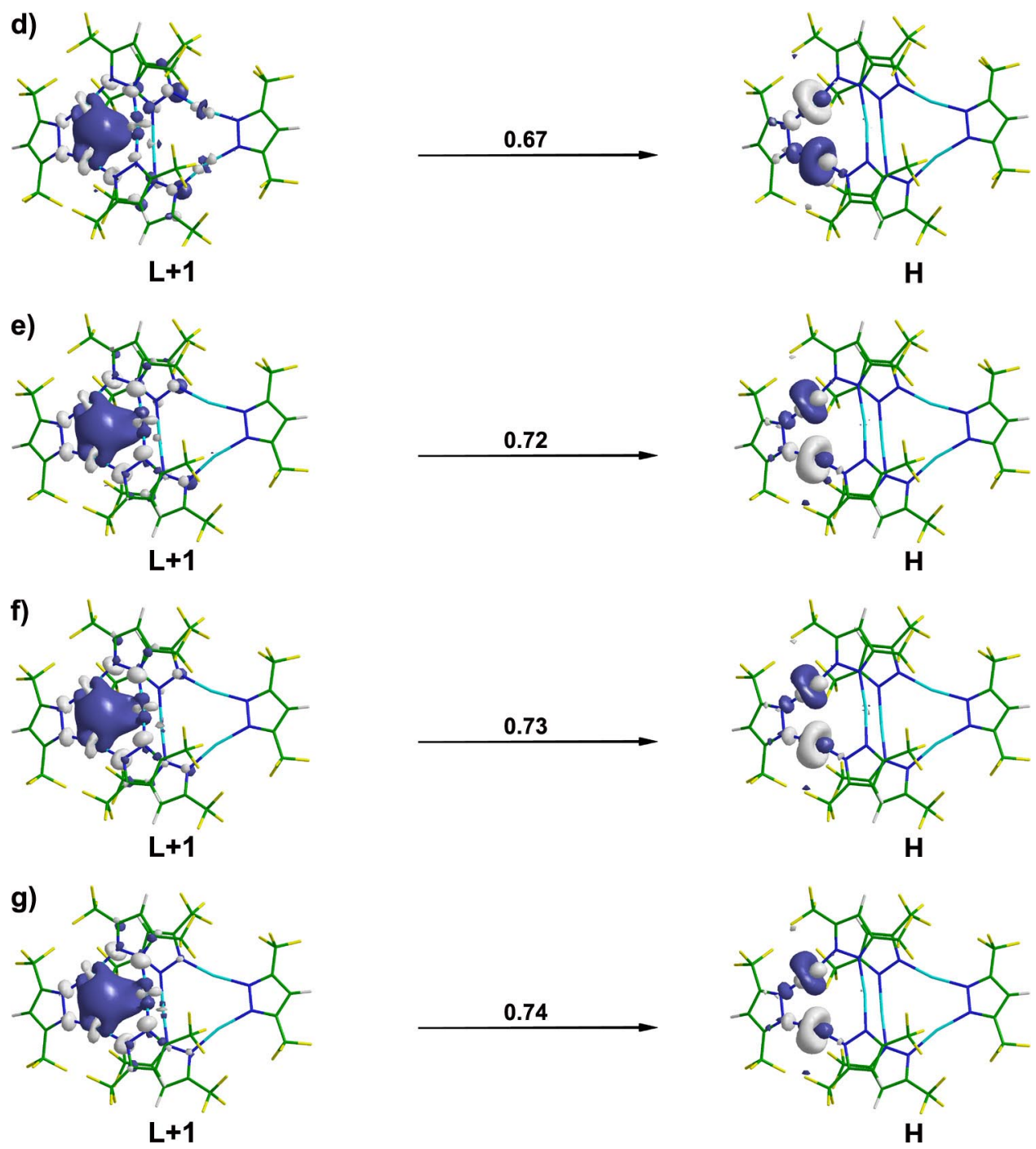

Figure S4. The TD-B3PW91/6-31G calculated triplet electron transition (illustrations) for a) $D_{t 1}$, b) $D_{t 2}$, c) $D_{t 3}$, d) $D_{t 4}$, e) $D_{t 5}$, f) $\left.D_{t 6}, g\right) D_{t 7}$. 